\title{
3D Vector Reconstruction of the Muscles of the Ventral Region of the Neck from Anatomical Sections of Korean Visible Human at the Paris Descartes Anatomy Laboratory
}

\author{
Abdoulaye Kanté1,2*, Jean François Uhl'3 , Mariam Daou²,4, Babou Ba², Tata Touré2, \\ Ousmane Touré ${ }^{2}$, Yatera Demba ${ }^{2}$, Moumouna Koné ${ }^{2}$, Drissa Traoré ${ }^{2}$, Nouhoum Ongoïba ${ }^{2}$, \\ Abdel Karim Koumaré2 \\ ${ }^{1}$ Anatomy Laboratory, University of Paris 5, Paris, France \\ ${ }^{2}$ Anatomy Laboratory, University of Sciences, Techniques and Technologies of Bamako, Bamako, Mali \\ ${ }^{3}$ URDIA Research Unit, Paris Descartes University, Paris, France \\ ${ }^{4}$ Department of Neurology, Gabriel Touré University Hospital in Bamako, Bamako, Mali \\ Email: *kanteim@yahoo.fr
}

How to cite this paper: Kanté, A., Uhl, J.F.., Daou, M., Ba, B., Touré, T., Touré, O., Demba, Y., Koné, M., Traoré, D., Ongoïba, N. and Koumaré, A.K. (2022) 3D Vector Reconstruction of the Muscles of the Ventral Region of the Neck from Anatomical Sections of Korean Visible Human at the Paris Descartes Anatomy Laboratory. Forensic Medicine and Anatomy Research, 10, 13-26.

https://doi.org/10.4236/fmar.2022.101003

Received: August 4, 2021

Accepted: December 28, 2021

Published: December 31, 2021

Copyright $\odot 2022$ by author(s) and Scientific Research Publishing Inc. This work is licensed under the Creative Commons Attribution International License (CC BY 4.0).

http://creativecommons.org/licenses/by/4.0/

(c) (i) Open Access

\begin{abstract}
Objective: To carry out a 3D vector reconstruction of the muscles of the ventral region of the neck from anatomical sections of the "Korean Visible Human" for educational purposes. Materials and Methods: The anatomical subject was a 33-year-old Korean man who died of leukemia. He was $164 \mathrm{~cm}$ tall and weighed $55 \mathrm{kgs}$. The anatomical sections were made in 2010 after an MRI and a CT scan. A special saw (cryomacrotome) made it possible to make cuts $0.2 \mathrm{~mm}$ thick on the frozen body, i.e. 5960 cuts. Sections numbered 1500 to 2000 (or 500 cuts covering the neck) were used for our study. A segmentation by manual contouring of each anatomical element of the anterior neck region was done using Winsurf version 3.5 software on a laptop PC running Windows 7 equipped with an 8 gigabyte RAM. Results: We modeled the sternocleidomastoid muscles, the supra-hyoid muscles, the infra-hyoid muscles and the muscle structures of the anterior neck region, the aero-digestive axis of the anterior neck region and the vasculo-nervous axis of the neck. This model is easily manipulated using the Acrobat 3Dpdf interface. Each item accessible in a menu can be displayed, hidden or made transparent, and 3D labels are available as well as educational menus for learning anatomy. This vector model has been integrated into the Diva3d virtual dissection table, a new educational tool used by universities and medical schools to learn anatomy. This model was also uploaded to the Sketchfab ${ }^{\circledR}$ website and 3D printed using an ENDER $^{\circledR}$ pro 3 printer. Conclusion: This original work constitutes a remarkable educational tool for the anatomical study of the anterior neck re-
\end{abstract}


gion and can also be used as a $3 \mathrm{D}$ atlas for simulation purposes for training in therapeutic gestures.

\section{Keywords}

Anterior Neck Region, Korean Visible Human, 3D Vector Modeling, Diva3d Virtual Dissection Table, 3D Printing, Sketchfab

\section{Introduction}

The "head-neck" assembly forms an anatomical and functional unit connected to the trunk via the neck. This is limited cranially by the caudal border of the mandible, the apex of the mastoid process and the external occipital protuberance and caudally by the jugular notch, clavicle, acromion and spinous process of the 7th cervical vertebra [1]. It contains numerous vasculo-nervous structures to which the cervical viscera are branched off [1]. The ventral side of the neck is separated into two regions by the hyoid bone, one supra hyoid and the other infra hyoid [2]. The neck muscles are classified according to their position relative to the cervical spine into lateral muscles, ventral muscles and back muscles and the ventral muscles are divided into supra-hyoid and infra-hyoid muscles [3].

We carried out this study in order to carry out a 3D reconstruction of the muscles of the ventral face of the neck for educational purposes.

\section{Methodology}

Our database was a series of anatomical sections of the Koran Visible Human $(\mathrm{KVH})$. The subject was a 33-year-old young adult of standard height: length $1.65 \mathrm{~m}$; weight $55 \mathrm{kgs}$. It was frozen at $70^{\circ} \mathrm{C}$. A cryomacrotome (Figure 1 (a) and Figure 1(b)) was used to cut the entire body into 8506 sections. The cross section of the body was made from the apex to the perineum at $0.2 \mathrm{~mm}$ intervals and from the perineum to the toes at $1 \mathrm{~mm}$ intervals. This produced 8506 anatomical
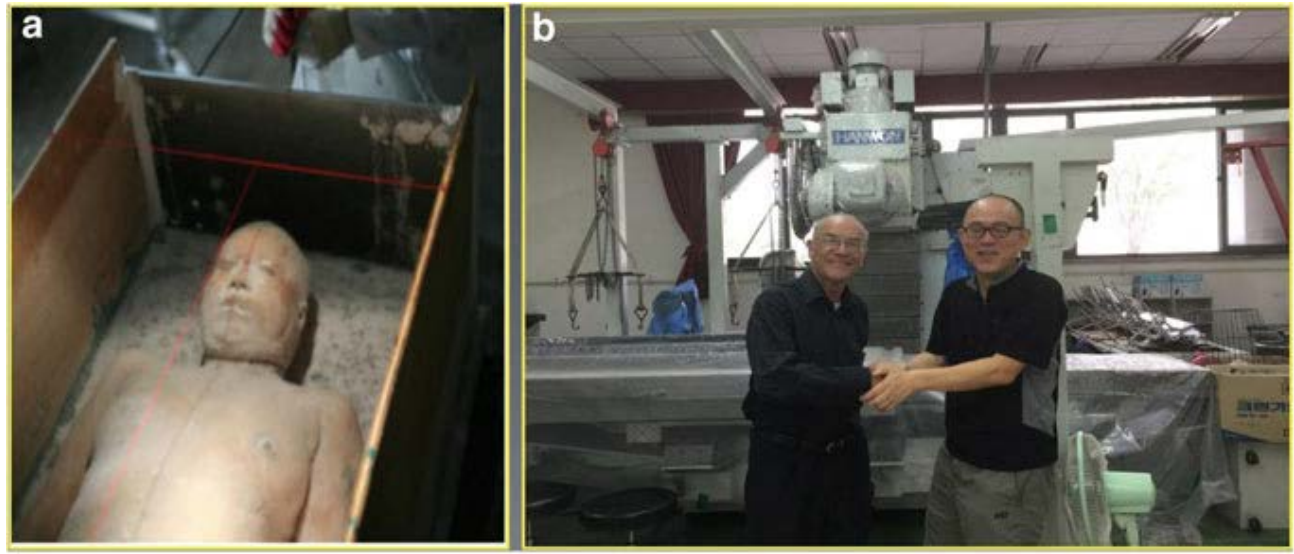

Figure 1. (a) The frozen body of the anatomical subject of KVH. (b) Cryomacrotome (Pr Chung, University of Ajou, Seoul, Korea on the right and Pr Uhl Université Paris Descarte). 
sections of the whole body (Park et al. 2005a). In order to photograph the severed body surfaces, a Canon MC EOS-1Ds Mark III $^{\mathrm{TM}}$ was used, fitted with a Canon $^{\mathrm{TM}}$ EF $50 \mathrm{~mm}$ f/1.2L USM lens. The resolution of the resulting images was $5616 \times 3744$ pixels, saved in TIFF format. Since 2010, the Korean team has carried out vectorial $3 \mathrm{D}$ reconstruction from this data with the following steps:

- The first step was the colored segmentation cut by cut using Photoshop. Each anatomical element was delimited with the magic wand (Park et al. 2005b).

- The second step was to create a 3D vector model with Mimics ${ }^{\circledR}$ from the series of colored segmented sections.

- The next steps were the integration of these licenses into the Maya ${ }^{\circledR}$ E3D model... Finally, the resulting 3D model has been converted to a ".u3d" file format in order to: take advantage of the Acrobat 3Dpdf interface, for an interactive display of 3D modeling with anatomical cuts. This enormous segmentation work has been carried out in 8 years by the Korean team. Our own methodology uses the same anatomical sections with the following five steps (Figure 2).

- Segmentation and 3D vector modeling of the muscles of the anterior region with Winsurf ${ }^{\circledR}$ software version 3.5 (Moody and Lozanoff 1997) from anatomical sections.

- Export of the Winsurf ${ }^{\circledR}$ mesh in cad format.

- Refinement of the mesh, cleaning and arrangement with Blender ${ }^{\circledR}$.

- Classification of the muscles of the anterior region with Acrobat 3D toolkit ${ }^{\circledR}$.

- Creation of the final interface with Acrobat pro ${ }^{\circledR}$.

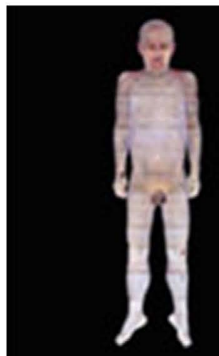

KVH

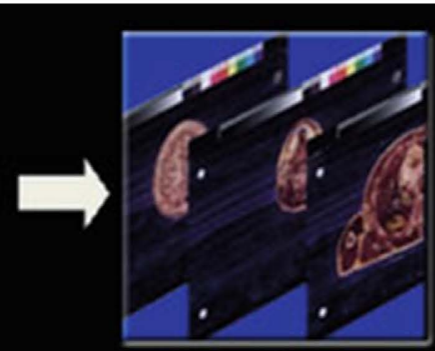

6000 slices

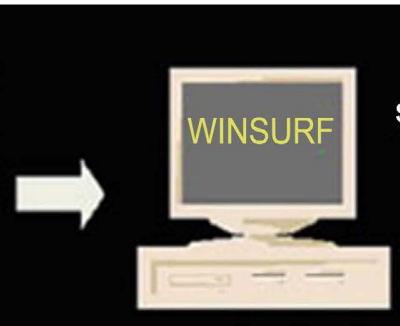

SEGMENTATION
1200 structures

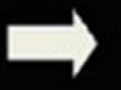

EXPORT in CAD format

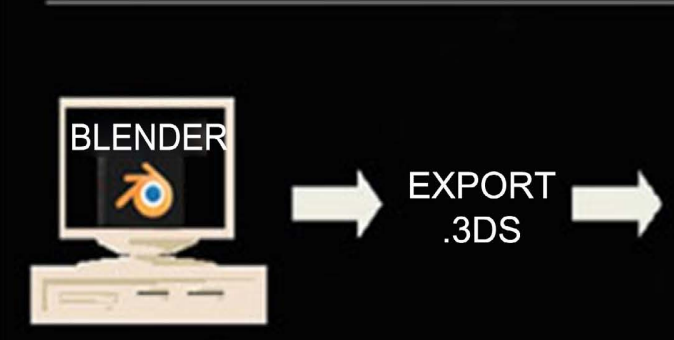

improve 3D mesh Names and coloring

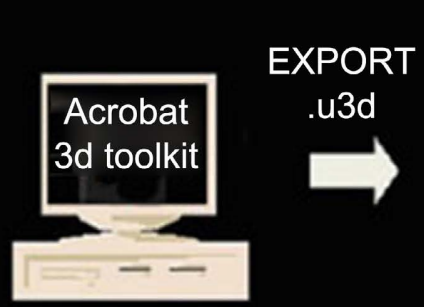

Hierarchy

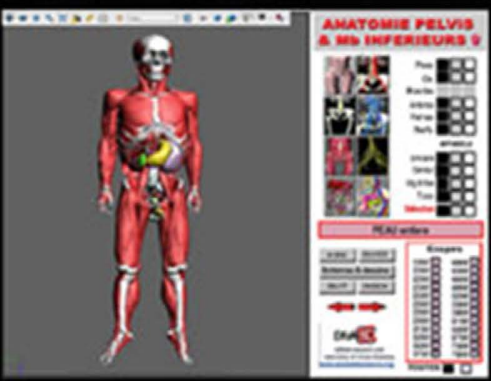

Javascript $>$ 3D-PDF/app

Figure 2. Methodology of anatomical sections to the Atlas of the final body. 


\subsection{Segmentation and 3D Reconstruction of Mesh Models}

We performed segmentation on anatomical sections of the neck of $\mathrm{KVH}$ data using Winsurf ${ }^{\circledR}$ software (Moody and Lozanoff, 1997).

\subsection{Creation of New 3D Objects}

Each 3D object representing an anatomical structure was constructed separately, identified by manually placing chains of dots around the edges of the object, in this way each distinct anatomical structure was segmented by mapping. Figure 3 shows the software interface, with the tools available at the top left side of the window to edit or modify the outlines. Here, the boundaries of the sternohyoid muscle have been drawn.

\subsection{Scale Measurement of the 3D Model}

The scale parameters were measured on a section as well as the section thickness by a special software function. The resulting value was $5650 / 2 / x$, where $\mathrm{x}$ represents the jump between slices during display. The same scale settings should be used for all anatomical objects. The creation of 3D anatomical objects is achieved by describing them section by section: this is a complete manual segmentation of the objects. This step may seem long and tedious, but it is an essential part of the process of learning anatomy. Once the contours were assigned to each serial section containing the object, the vertices were connected using a surfacing routine specially developed for the Winsurf ${ }^{\circledR}$ software (Uhl et al., 2006). This routine calculates and refines an optimal solution to assemble the vertices into an optimized volumetric 3D object. Figure 4 shows how to use multiple color channels to construct the thoracic limb vein tree. This option is very useful for the user because it allows locating an error or missing information on one of the colored channels. Once completed, all the objects could be put

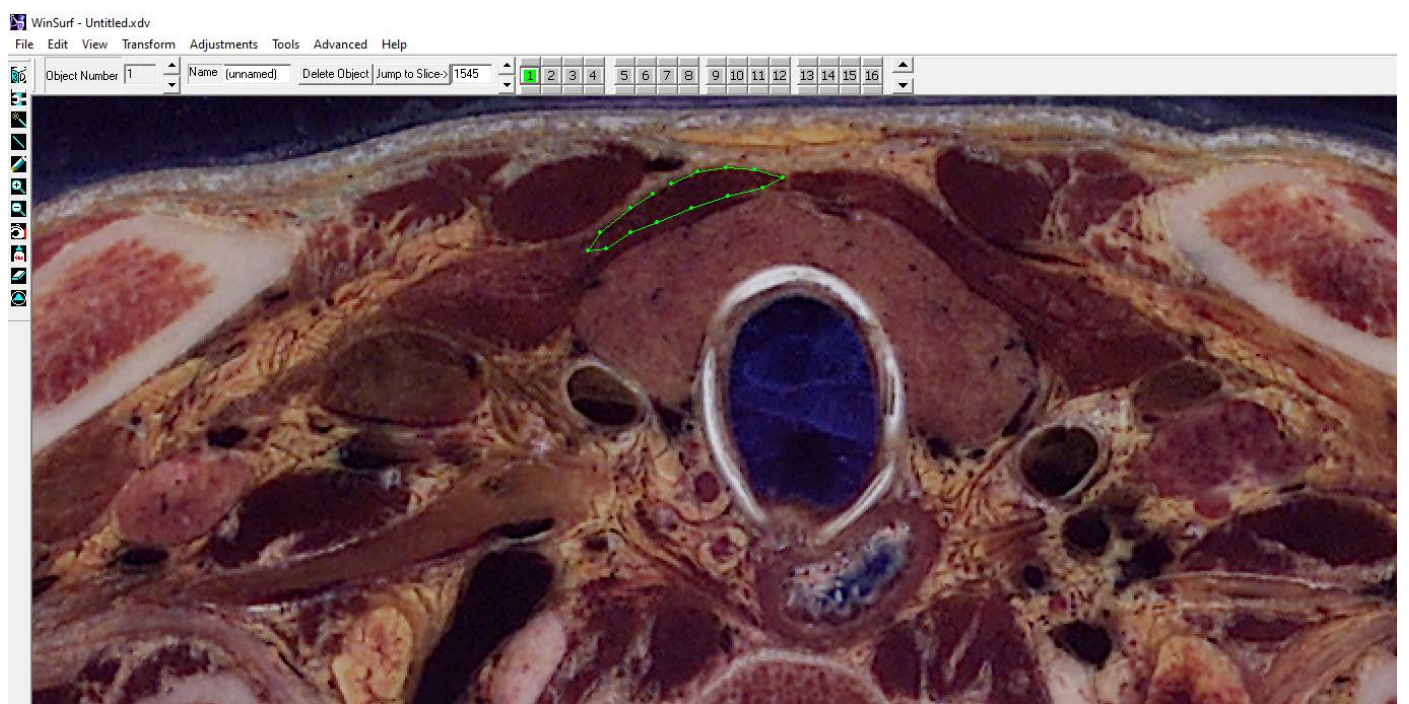

Figure 3. Creation of a $3 \mathrm{D}$ mesh from the $2 \mathrm{D}$ section of the right sternohyoid muscle with the Winsurf ${ }^{\mathrm{B}}$ software using a green channel. 


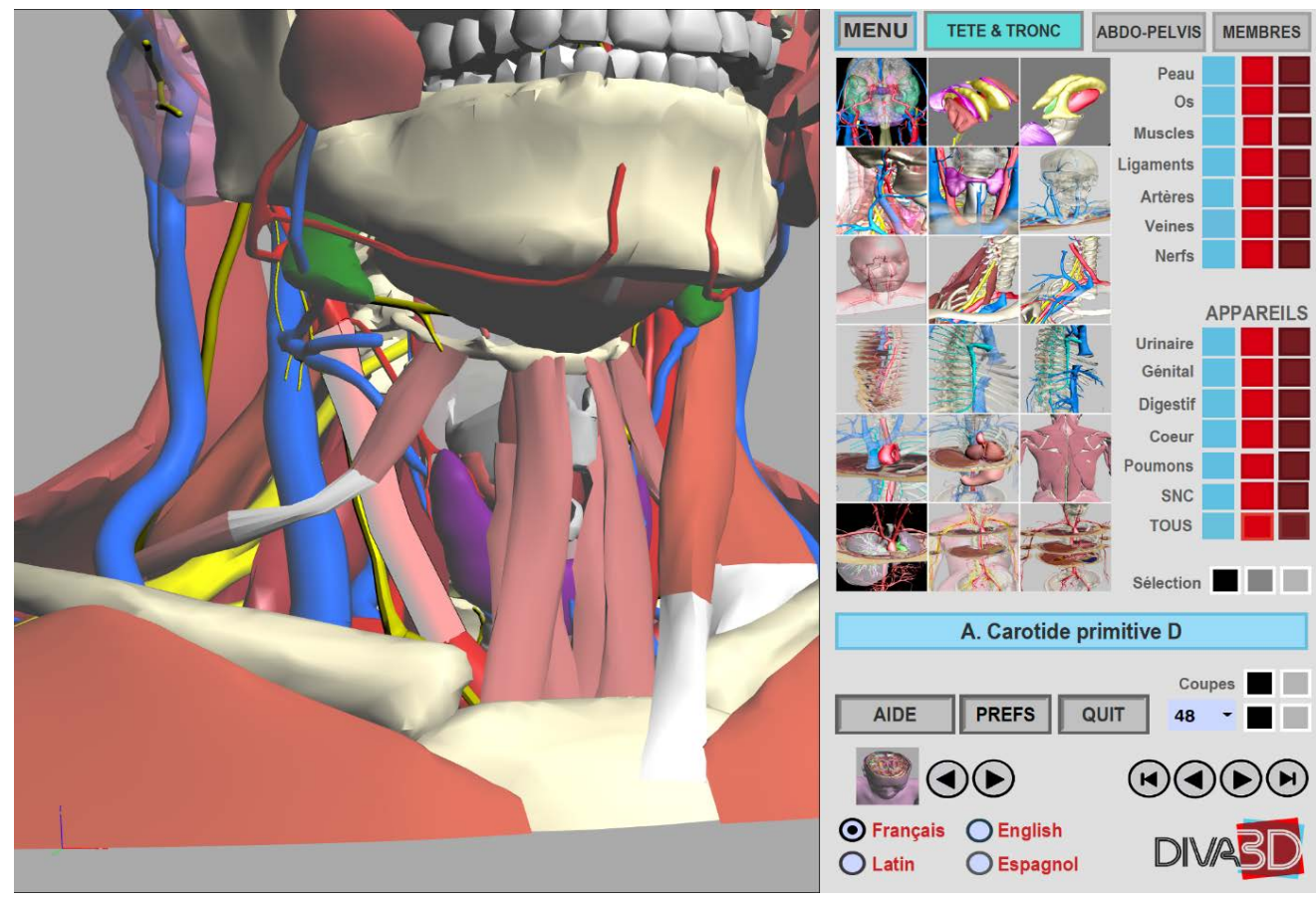

Figure 4. Virtual dissection board interface screen showing the 3D model of the ventral region of the right neck. On the left, the 3D window. On the right, the function buttons to choose the display mode to be selected by zone, device, system, organ and/or sections.

together using the software's "add surface file" option, in order to get the final 3D model. In addition, textures could be applied to the objects in order to obtain a more realistic 3D reconstruction.

\subsection{Export of the 3D Mesh Model Produced by Winsurf ${ }^{\circledR}$ Version 3.5}

The second step was to export the entire 3D vector model built by Winsurf ${ }^{\circledR}$ to $3 \mathrm{D}$ PDF format in order to improve the anatomical model. In fact, Winsurf ${ }^{\circledR}$ is old, inexpensive software that works well with the old Windows ${ }^{\circledR}$ XP operating system and is easy to use. Before this export, it is important to make sure that each anatomical object has a different RGB color, in order to import it as a separate anatomical element in the Acrobat format. The format conversion was performed using a simple function of Adobe Acrobat ${ }^{\circledR} 3 \mathrm{D}$ version 9, in the software menu option: "Create PDF file by 3D capture". It can then be exported as .wrl and exported to Blender software.

\subsection{Mesh Refinement with a Powerful Free Modeler: Blender ${ }^{\circledR}$ v 2.79b (http://blender.com)}

The improvement of the mesh models and the correction of some anatomical problems were carried out with Blender ${ }^{\circledR}$, as well as the integration of a series of 100 anatomical sections into the $3 \mathrm{D}$ model. This step is very important to improve model quality and anatomical accuracy, as well as to improve understanding of anatomical relationships in 3D space. 


\subsection{Exporting the 3D Model in Acrobat 3D Toolkit ${ }^{\circledR}$}

After exporting the entire 3D Blender model and sections in .obj or .3Ds format, Acrobat 3D Toolkit ${ }^{\circledR}$ software was used to produce a u3d compatible file and to configure the hierarchical list of anatomical elements, display them in the model tree window located to the left of the Acrobat window.

\subsection{D Interactive Display with Acrobat ${ }^{\circledR}$ 3D PDF Interface}

The interface is very versatile with the control panel on the right, giving access to the different anatomical regions of interest, here the ventral region of the neck (Figure 4). A selection of the interactive 3D model is possible:

- by zone (full body, trunk, head and neck, thoracic limbs, pelvic limb icons at the top)

- subsystem (whole, urinary, genital, food, respiratory, circulatory)

- by region of interest (pelvis, neck, brain, hand, foot, etc.)

For any of these options, by clicking on the colored boxes in this list of anatomical structures (on the right), you can make any element visible, hidden or transparent. You can also select and display horizontal sections with 3D models (Figure 5). Other functions of the Acrobat ${ }^{\circledR}$ interface are also available using the $3 \mathrm{D}$ toolbar located at the top of the display window (Figure 5). Menu of interactive manipulation functions of the 3D model, choice of lighting effects, background color, 3D rendering options (solid, wireframe, shaded wireframe, transparent wireframe, illustration ...), 3D labels, cross section in any plan, and
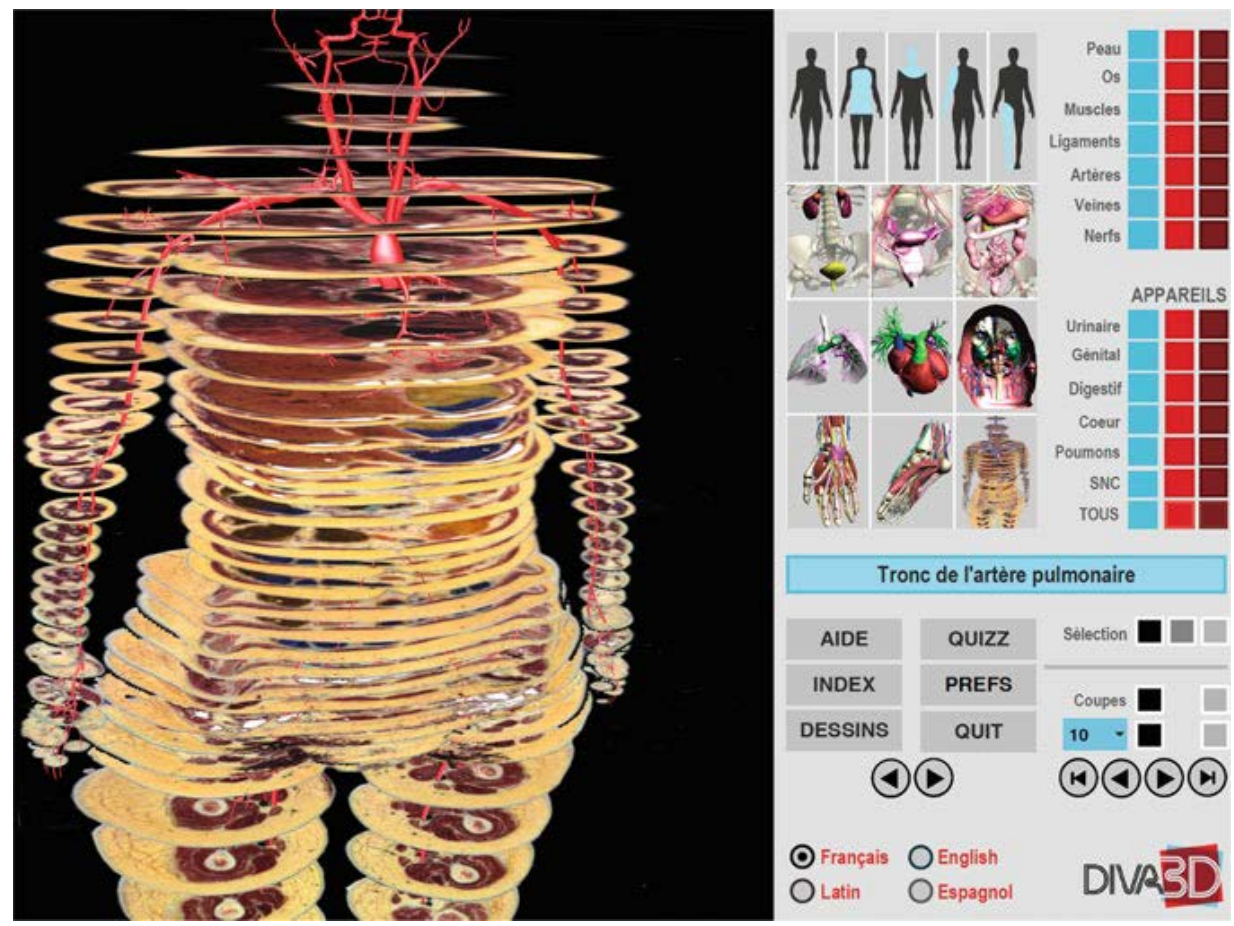

Figure 5. Virtual dissection board interface screen showing the 3D model with the anatomical cuts (in French). The 3D window on the left shows the display of anatomical sections original with the vector models (here the arteries). 
"menu" memorize the display view with all the parameters of the 3D anatomical model.

\section{Results}

Our methodology allowed us to perform the 3D reconstruction of all the muscles of the ventral region of the neck (Figure 6).

Sterno-cleido-mastoid muscle (Figure 7 \& Figure 8)

Supra-hyoid muscles (Figures 9-13)

3D reconstruction of the infra-hyoid muscles (Figures 14-19)

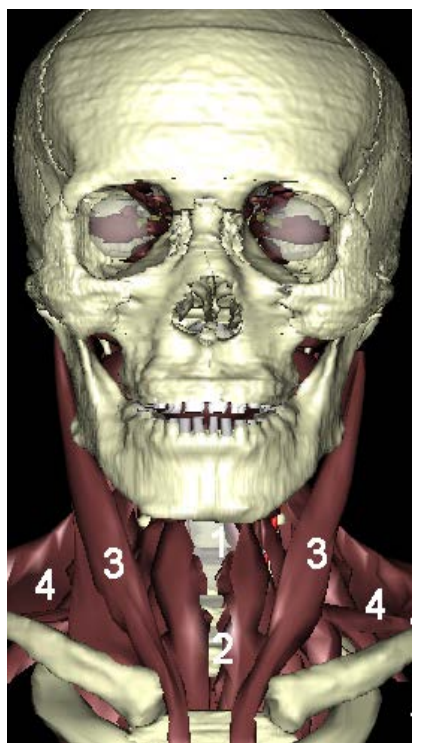

Figure 6. Ventral view of the muscles and muscle regions of the neck. 1. Supra-hyoid region; 2. Infra-hyoid region; 3. Carotid region; 4. Supraclavicular region.

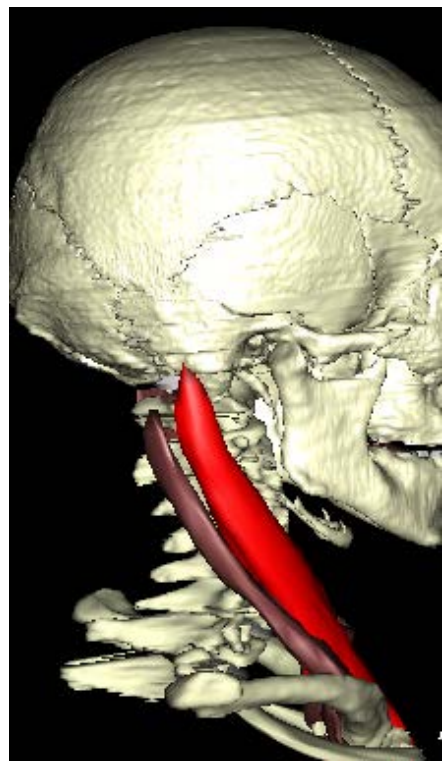

Figure 7. Sterno-cleido-mastoid muscle. Sterno-mastoid leader colored in red. Right side view. 


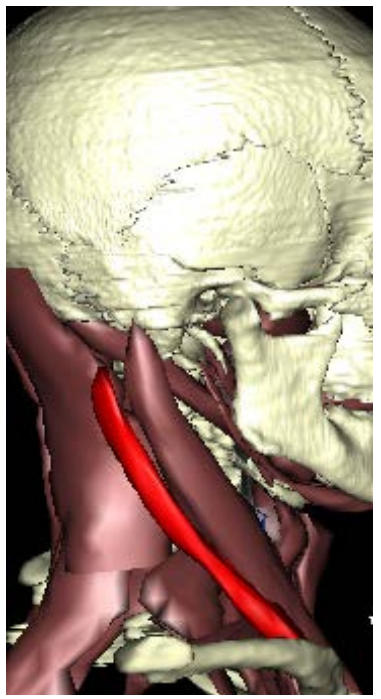

Figure 8. Right lateral view of the neck muscles. Clavicular or sterno -occipital head colored red.

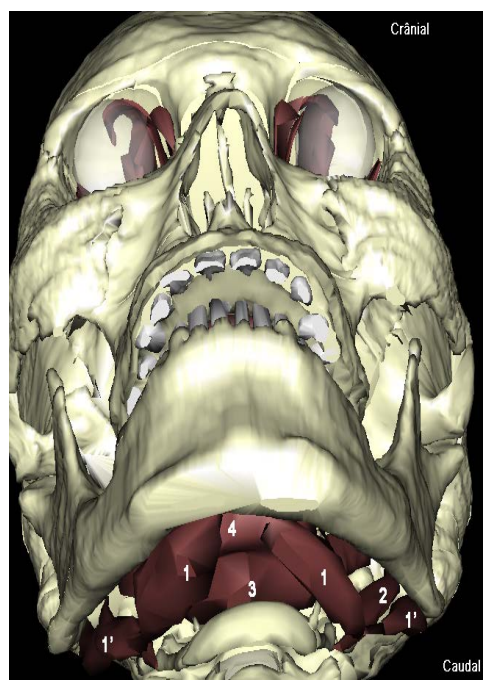

Figure 9. Supra-hyoid muscles. 1. Digastric muscle (1. Anterior belly; 1'. Posterior belly); 2. Stylohyoid muscle; 3. Geniohyoid muscle; 4 . Mylohyoid muscle.

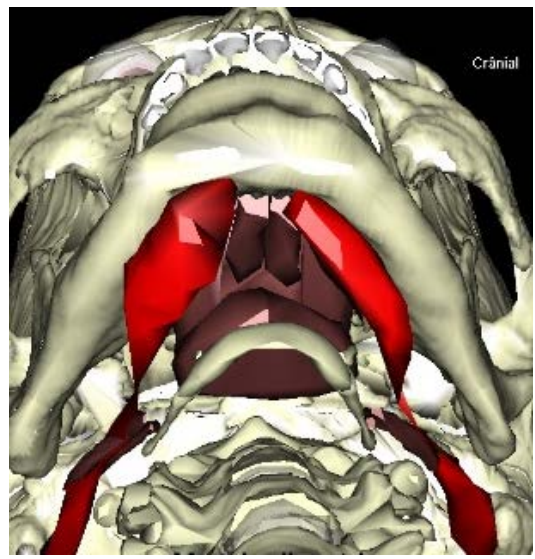

Figure 10. Digastric muscle. 


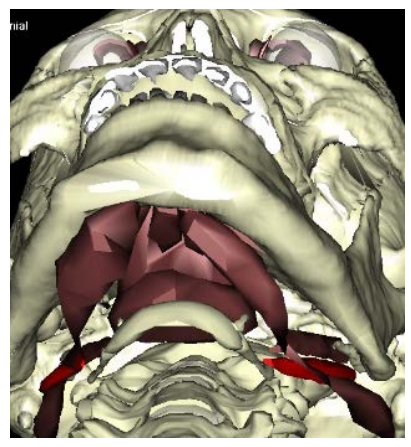

Figure 11. Stylohyoid muscle.

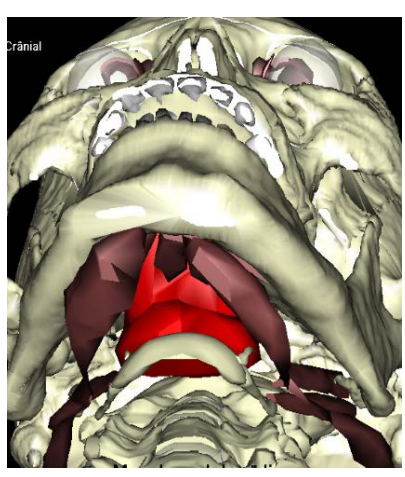

Figure 12. Mylohyoid muscle.

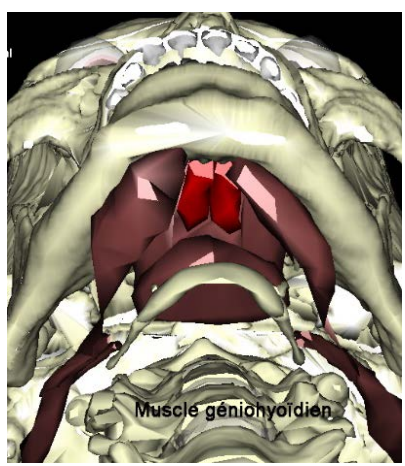

Figure 13. Geniohyoid muscle.

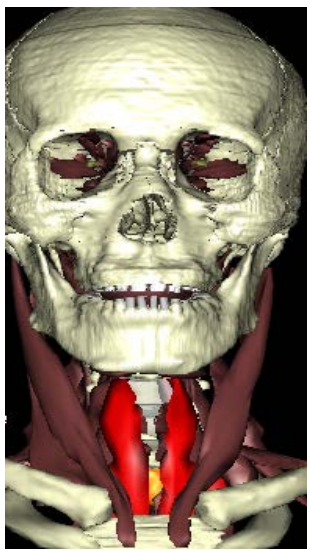

Figure 14. Ventral surface of the neck muscles. Sternohyoid muscle colored red. 


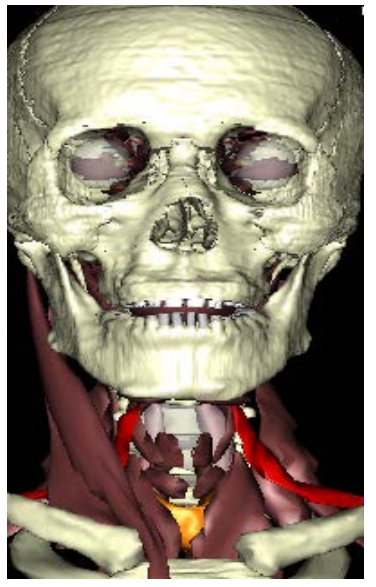

Figure 15. Ventral surface of the neck muscles. Omohyoid muscle colored red.

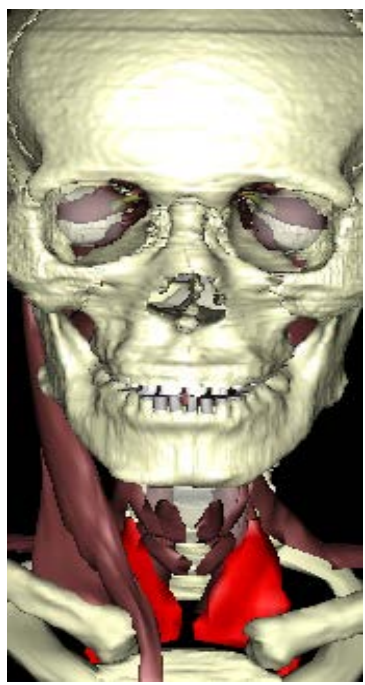

Figure 16. Ventral surface of the neck muscles. Sterno-thyroid muscle colored red.

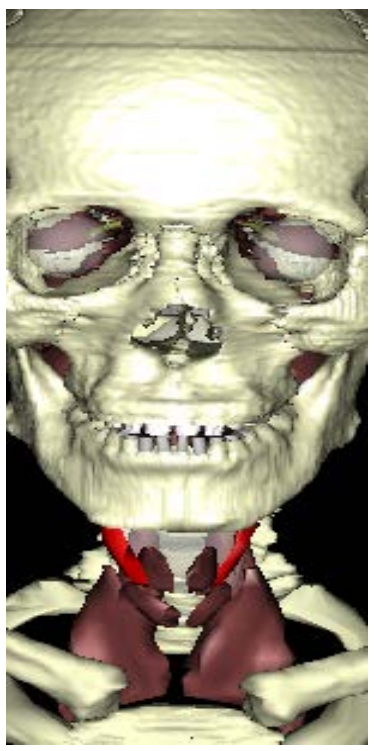

Figure 17. Ventral surface of the neck muscles. Thyro-hyoid muscle colored red. 


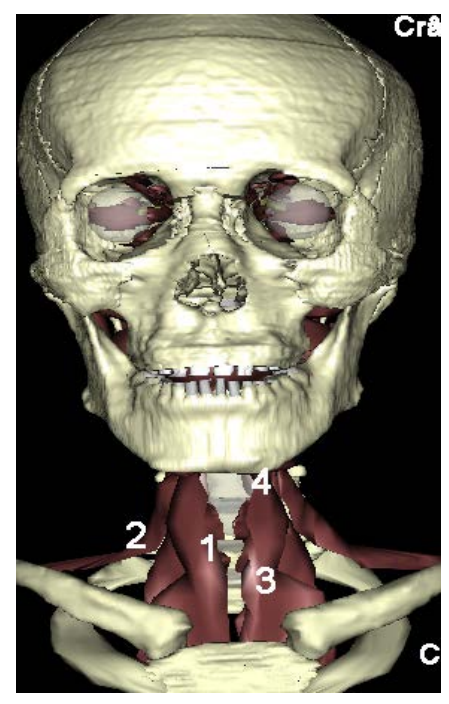

Figure 18. Infra-hyoid muscles. 1. Sternohyoid muscle; 2. Omohyoid muscle; 3. Sterno-thyroid muscle; 4 . Thyrohyoid muscle.
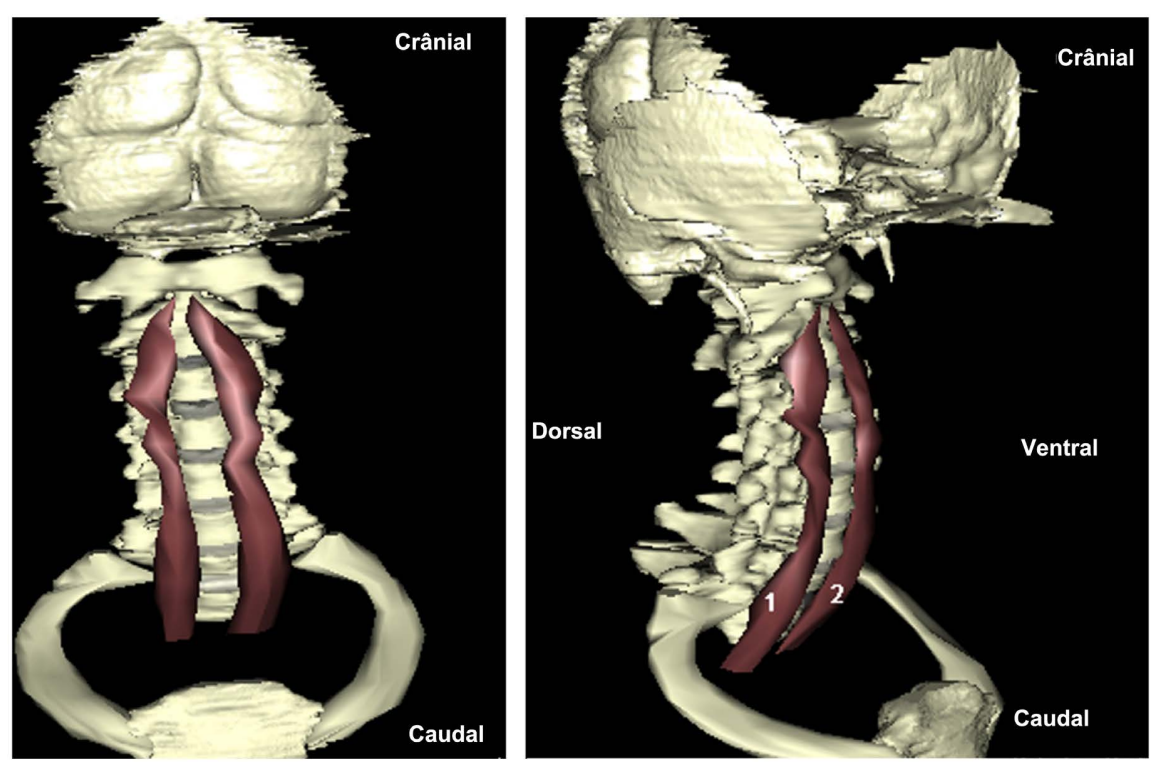

Figure 19. Long muscles of the neck: ventral view on the left; right side view. 1. Long muscle of the right neck; 2. Long muscle of the left neck.

Our 3D vector reconstruction of the muscles of the ventral neck region was integrated into the DIVA3D virtual dissection table (Figure 20). It was also posted on the Sketchfab website (Figures 21-23).

\section{Discussion}

The advantage of our 3D vector modeling of the muscles of the ventral region of the neck is essentially based on the fact that all of the contouring work and therefore all of the 3D reconstruction was carried out from real sections of the human body. The result is a major increase in the precision and reliability inherent in the results presented above. 


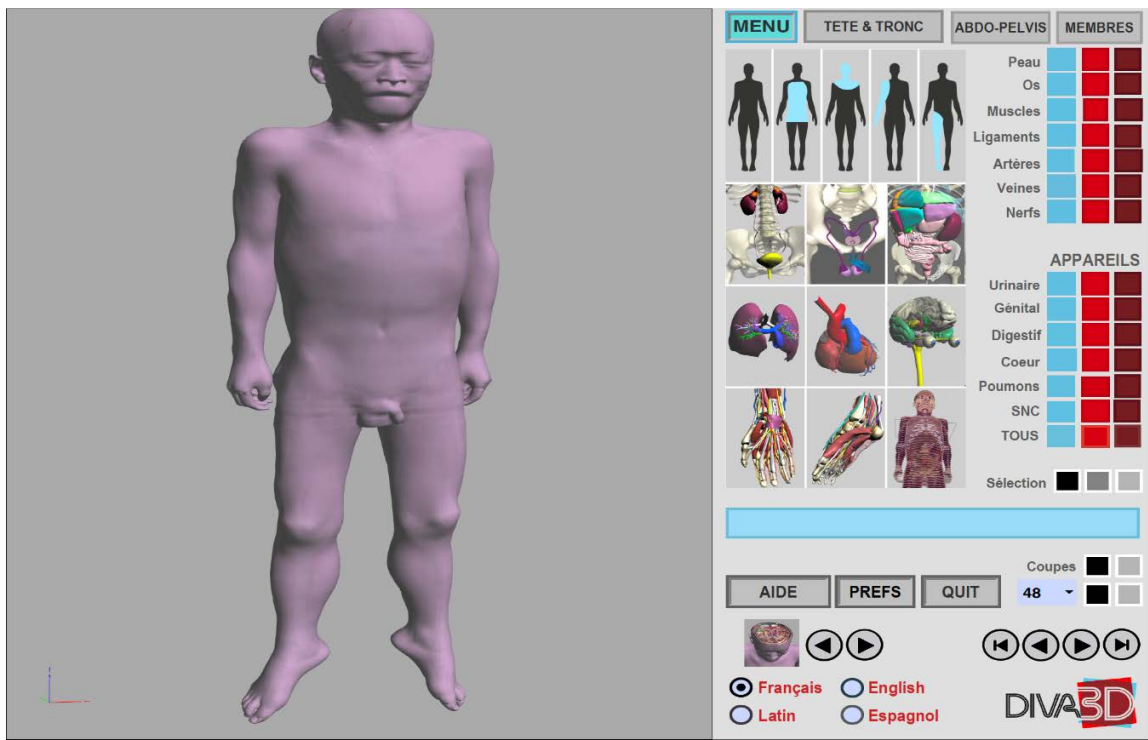

Figure 20. Virtual dissection table interface screen. On the left the $3 \mathrm{D}$ window. On the right, the function buttons to choose the display and the mode to be selected by zone, device, system, organ and/or unit.

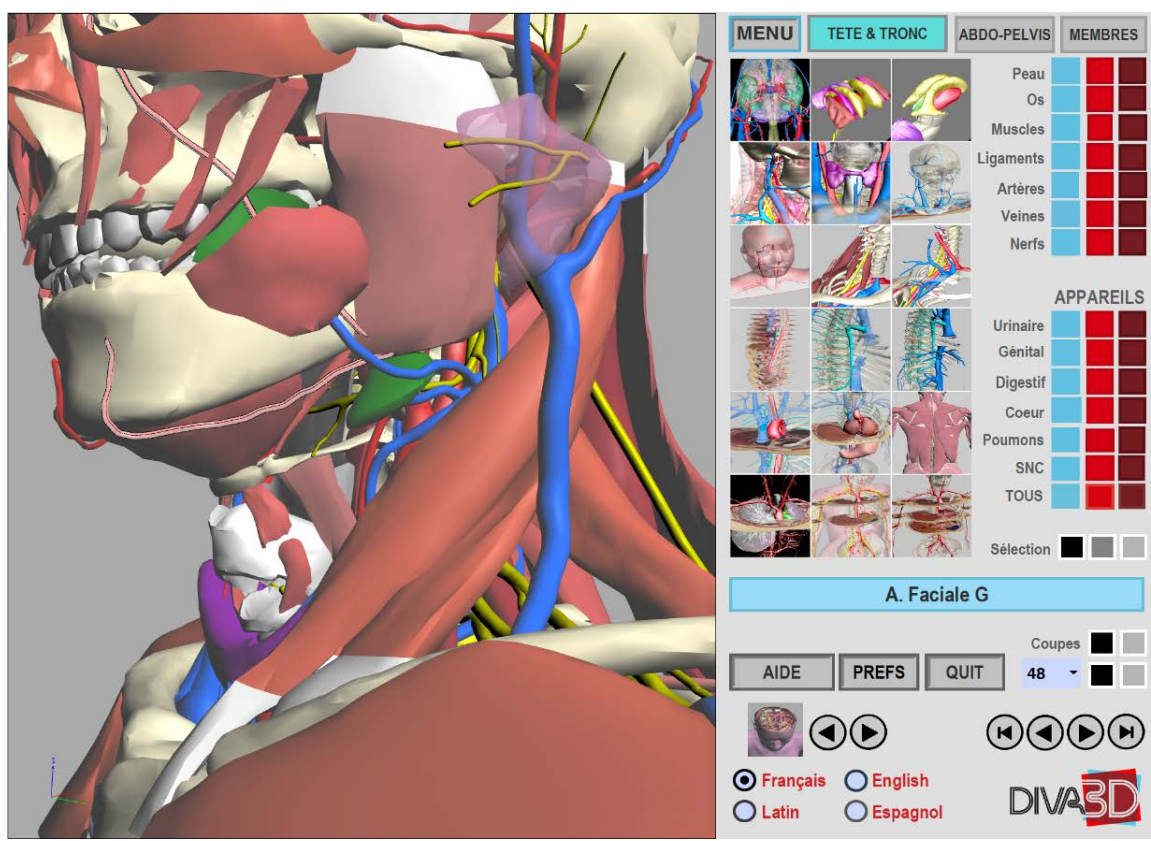

Figure 21. Virtual dissection table interface screen highlighting the sternocleidomastoid muscle. On the left the 3D window. On the right, the function buttons to choose the display and the mode to be selected by zone, device, system, organ and/or unit.

Indeed, automatic reconstructions of the muscles of the ventral region of the neck using digital methods such as MRI and CT scan can prove to be somewhat disappointing in the sense that certain bone structures are missing. In contrast to this process, this contouring work relies on manual segmentation, which involves anatomical expertise, which reduces the risk of errors in reconstruction. The second advantage is based on the fact that better precision as well as the 


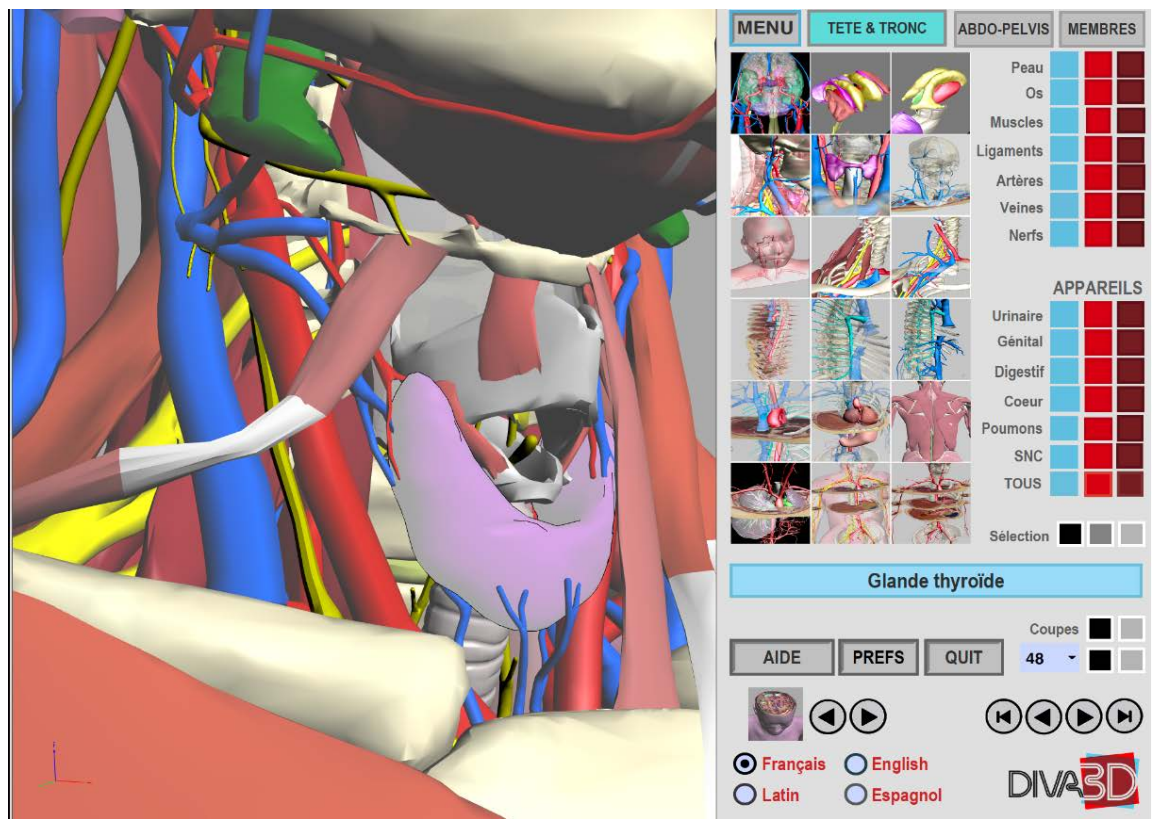

Figure 22. Virtual dissection table interface screen showing the digastric muscle. On the left the $3 \mathrm{D}$ window. On the right, the function buttons to choose the display and the mode to be selected by zone, device, system, organ and/or unit.
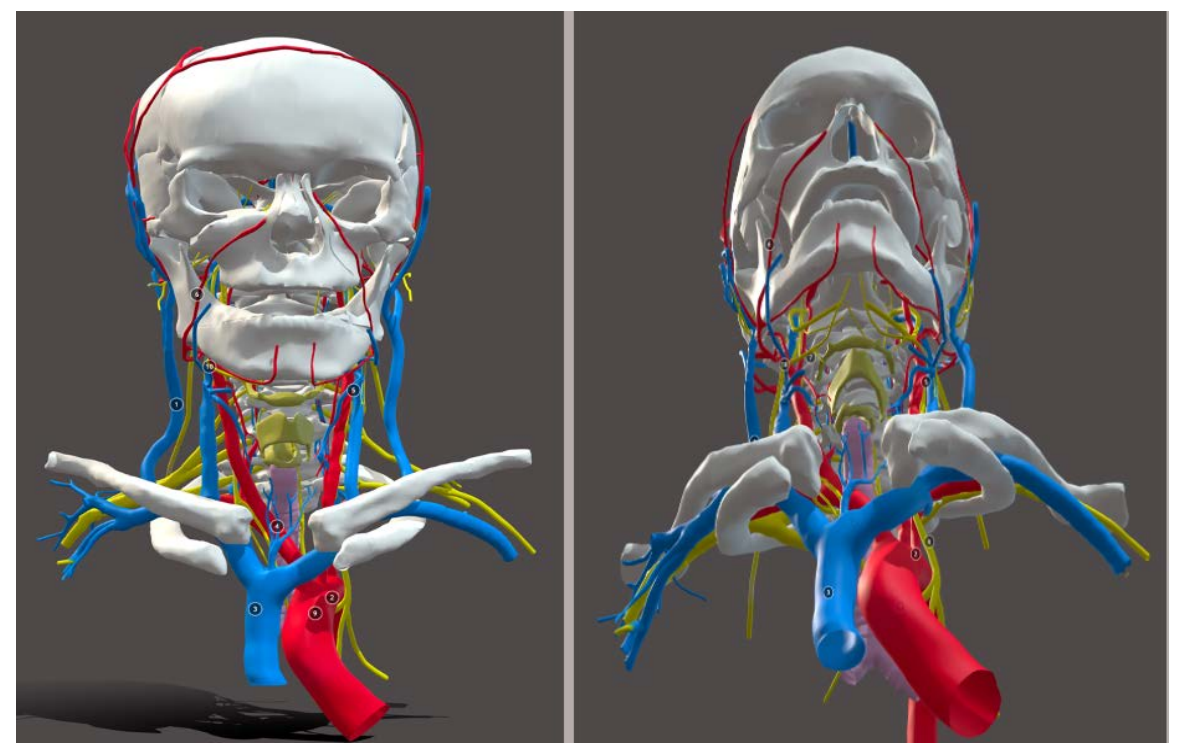

Figure 23. 3D model of KVH's neck and head displayed with Sketchfab software Available at: https://skfb.ly/6QYyo (Anterior right view and lower left view.

possibility of individualization of the different bone structures of the neck favors massive application in the academic field, thus contributing to a better understanding by students of medicine and other fields. In addition, it is fundamental to underline that this application is not restricted to the university field but can also be the support of a "surgical training" thus allowing a continuous training of the surgeons and a fortiori an improvement of their aptitude in their daily practices. 
In addition, the anatomical slices are of very high resolution unlike those of the Korean team working on segmented slices.

In addition, the cutting intervals were relatively small $(0.2 \mathrm{~mm}$ vs $1.0 \mathrm{~mm}$ in the KVH) which greatly facilitate our contouring work. Finally, it is clear that "Winsurf" and Acrobat 3D PDF are particularly easy to learn software, which is not the case with other 3D modeling and manual segmentation software. In addition, they offer fairly wide ranges of textures which further increases the realism that can be brought to our final work. Our 3D modeling of the muscles of the ventral region of the neck clearly testifies to the technological advances undertaken in the medical and scientific field in general. It is an outstanding tool for teaching ventral neck myology in medical science faculties.

\section{Conclusion}

Our 3D vector reconstruction of the muscles of the ventral region of the neck from the anatomical sections of Korean Visible Human is an original educational tool that easily allows teaching the myology of the ventral face of the neck and can also be the support of a "Surgical training" thus allowing a continuous training of the surgeons and an improvement of their aptitude in their daily practices.

\section{Acknowledgements}

We would like to thank Mr. Park and Mr. Chung for making the anatomical sections of $\mathrm{KVH}$ available to us.

\section{Ethics Commission}

We have obtained the consent of the Ethics Committee to write this article.

\section{Conflicts of Interest}

The authors declare no conflicts of interest regarding the publication of this paper.

\section{References}

[1] Schunke, M., Schulte, E., Schumacher, U., Voll, M. and Wesker, K. (2016) Head, Neck and Neck Fasciae. Prometheus Anatomy Atlas: Head, Neck and Neuroanatomy. Deboeck D Superior, Paris, 4.

[2] Bargy, F. and Beaudoin, S. (2010) Regions. In: The Keys to Anatomy, Ellipses, Paris, 172.

[3] Trost, O. and Trouilloud, P. (2011) The Muscles of the Neck. In: Head, Neck, Cranial Nerves and Sense Organs Anatomy, Ellipses, Paris, 83. 